\title{
Electron-Positron Momentum Densities for Electrons from Individual Core Levels
}

\author{
A. RubaszeK \\ W. Trzebiatowski Institute of Low Temperatures and Structure Research \\ Polish Academy of Sciences, 50-950 Wrocław 2, P.O. Box 1410, Poland \\ In the present contribution I focus on various effects affecting the slope of \\ calculated electron-positron momentum densities for electrons from individ- \\ ual core shells. In particular, I discuss the influence of positron distribution, \\ of approximation used for the electron-positron correlation functions, as well \\ as of finite apparatus resolution on the resulting spectra.
}

PACS numbers: 78.70.Bj, 71.20.--b, 71.60.+z

\section{Introduction}

Recent experiments demonstrate that the application of positron annihilation induced Auger electron spectroscopy combined with Doppler broadening technique makes it possible to map the electron-positron (e-p) momentum densities for electrons from individual core shells [1]. The Doppler spectra from core electrons may be interpreted as one-dimensional (1D) projections of the relevant e-p momentum densities, i.e.

$$
N_{n l}\left(p_{z}\right)=\int \mathrm{d} p_{x} \int \mathrm{d} p_{y} \rho_{n l}(\boldsymbol{p})=2 \pi \int_{\left|p_{z}\right|}^{\infty} p \rho_{n l}(|\boldsymbol{p}|) \mathrm{d} p,
$$

where the e-p density distribution for individual core shells, $n l$, reads as

$$
\rho_{n l}(\boldsymbol{p})=\rho_{n l}(|\boldsymbol{p}|)=\sum_{j}\left|\int \mathrm{e}^{-\mathrm{i} \boldsymbol{p} \cdot \boldsymbol{r}} \psi_{n l j}(\boldsymbol{r}) \psi_{+}(\boldsymbol{r}) \sqrt{\gamma(\boldsymbol{r})} \mathrm{d} \boldsymbol{r}\right|^{2} .
$$

Here $\boldsymbol{p}$ is the momentum of the pair, $\psi_{+}$and $\psi_{n l j}$ stand for the positron and electron wave functions, respectively, and $\gamma(\boldsymbol{r})$ denotes the e-p correlation function. Within the independent particles model (IPM) $\gamma(\boldsymbol{r})$ is set equal to unity. The total core density is a sum of contributions from occupied shells.

In practice, discrepancies between measured and calculated spectra have been found for $\mathrm{Cu} 3 p$ and $\mathrm{Ag} 4 p$ core states [1]. This fact was attributed by the 
authors to likely mixed s-p positron state at the surface and approximation used for $\gamma(\boldsymbol{r})$ in Eq. (2).

\section{Calculation and results}

In the present work the influence of three various effects on resulting 1D spectra for individual core shells is studied on the example of gold, a $5 d$ electron metal of fcc structure. Specifically, in Figs. 1, 2, and 3 relative changes in $N\left(p_{z}\right)$ due to, respectively, e-p correlation effects, positron distribution, and finite resolution of apparatus are presented. As the reference levels the IPM e-p momentum densities, shown in Fig. 4, have been chosen. The electron wave functions, $\psi_{n l j}$, have been calculated relativistically, with the potential obtained from linear muffin-tin orbital (LMTO) method in the atomic spheres approximation (ASA) [2]. The e-p correlation functions were incorporated in Eq. (2) non-locally, within the weighted density approximation (WDA) [3]. The positron wave function was calculated using the LMTO ASA code in two ways: within the IPM and including WDA e-p correlation potential.

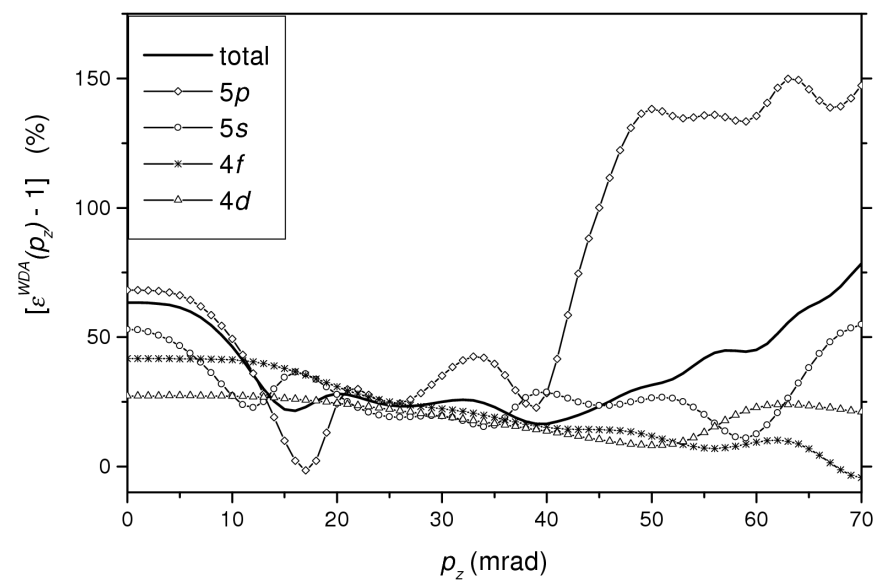

Fig. 1. Relative changes in $N\left(p_{z}\right)$ due to the non-local (WDA) e-p correlation effects. Here $\varepsilon^{\mathrm{WDA}}\left(p_{z}\right)-1=\left[N^{\mathrm{WDA}}\left(p_{z}\right)-N^{\mathrm{IPM}}\left(p_{z}\right)\right] / N^{\mathrm{IPM}}\left(p_{z}\right)$.

It can be seen in Fig. 4 that in the low momentum region (up to $15 \mathrm{mrad}$ ) as well as for momenta higher than $60 \mathrm{mrad}$, the $5 p$ electrons give the main contribution to $N^{\mathrm{IPM}}\left(p_{z}\right)$. In the remaining region $4 f$ electrons dominate in the spectrum. In the slope of densities shown in Fig. 4 the shoulders are visible. These shoulders are mostly pronounced for $5 p$ and $5 s$ electrons and they are hardly observed for $4 f$ electrons.

The first thing to note when comparing Figs. 1-3 is that the most considerable effect in $N\left(p_{z}\right)$ is due to the e-p interaction. As can be seen in Fig. 1, the 


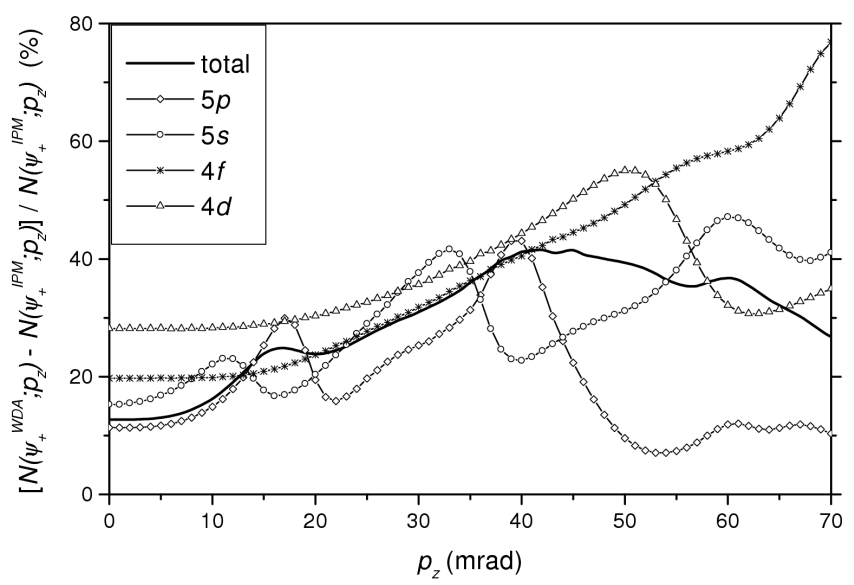

Fig. 2. Relative changes in $N^{\mathrm{IPM}}\left(p_{z}\right)$ due to the positron wave finction.

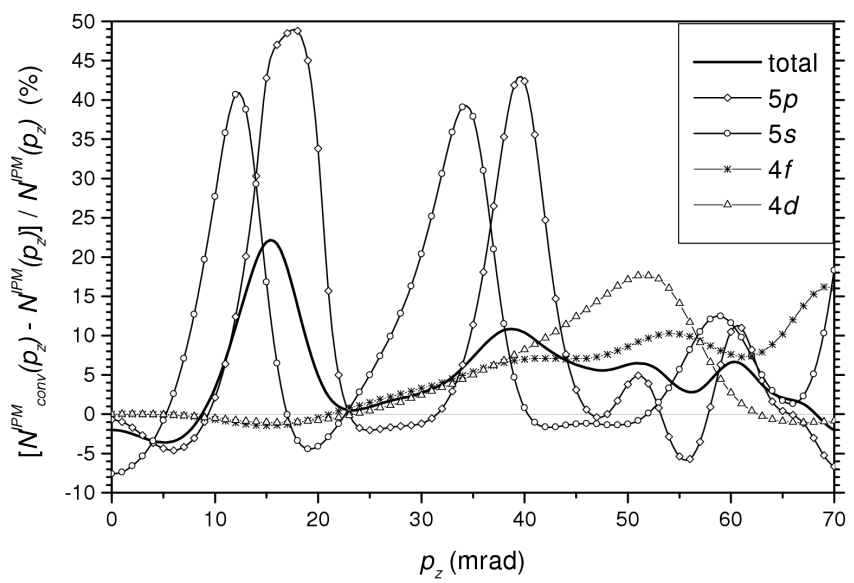

Fig. 3. Relative changes in $N^{\mathrm{IPM}}\left(p_{z}\right)$ due to the finite apparatus resolution. $N_{\text {conv }}^{\mathrm{IPM}}\left(p_{z}\right)$ was obtained by convolution of $N^{\mathrm{IPM}}\left(p_{z}\right)$ with Gaussian. The full width at half maximum of Gaussian is equal to $1.23 \mathrm{keV}$ [1].

relative change in $N^{\mathrm{IPM}}\left(p_{z}\right)$ exceeds $150 \%$ for $5 p$ electrons in the high momentum region, while for momenta close to zero these changes are greater than $25 \%$ for all electrons under study. It is also worth to point out the de-enhancement that occurs for $5 p$ electrons (for momenta close to $18 \mathrm{mrad}$ ) and $4 f$ electrons (for momenta close to $70 \mathrm{mrad}$ ). The possible explanation is oscillatory character of spherical Bessel functions, contributing to Eq. (2). Relative changes, due to including the $\mathrm{e}-\mathrm{p}$ correlation potential to the positron model are always positive (see Fig. 2). Indeed, "dressed" positron is less repulsive to ionic cores and therefore shifted towards the nuclei, as compared to its IPM counterpart. As a result, the overlap of positron and core electrons' wave function increases. This effect is not 


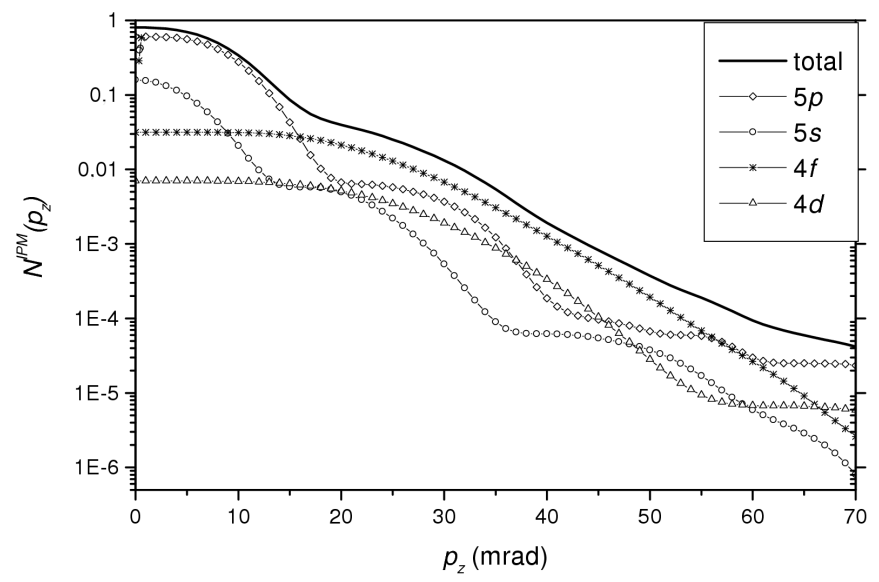

Fig. 4. 1D e-p momentum densities, $N^{\mathrm{IPM}}\left(p_{z}\right)$, calculated within the IPM for Au core shells.

negligible and it is well observed in the high momentum region for e.g. $4 f$ and $4 d$ electrons. Finally, the apparatus resolution shows the weakest influence on $N^{\mathrm{IPM}}\left(p_{z}\right)$, as presented in Fig. 3. Here relative changes do not exceed $10 \%$ for all electrons under study, in spite of rather poor resolution (of order of $5 \mathrm{mrad}$ ), assumed in the present calculation. The only exceptions are regions, where shoulders in $N^{\mathrm{IPM}}\left(p_{z}\right)$ have arms. For these momenta high humps in difference curves are seen, especially for $5 p$ and $5 s$ electrons (and in the relevant total density). In these regions convolution with Gaussian considerably smoothes out the negative slope of 1D momentum density and slightly shifts positions of arms.

\section{Summary}

Concluding, in studies of core contributions to the e-p momentum density incorporating the resolution function of apparatus hardly influences calculated spectra. It is not so for positron model and approximation used for e-p correlation functions, which both appreciably change calculated core spectra. Here an adequate treatment of these effects is highly desired.

\section{References}

[1] A. Eshed, S. Goktepeli, A.R. Koymen, S. Kim, W.C. Chen, D.J. O'Kelly, P.A. Sterne, A.H. Weiss, Phys. Rev. Lett. 89, 075503 (2002); S. Kim, N.G. Fazleev, A.R. Koymen, A. Eshed, S. Goktepeili, A.H. Weiss, Mater. Sci. Forum 445-446, 483 (2004).

[2] O.K. Andersen, Phys. Rev. B 12, 3060 (1975); W.R.L. Lambrecht, O.K. Andersen, ibid. 34, 2439 (1986).

[3] A. Rubaszek, Z. Szotek, W.M. Temmerman, Phys. Rev. B 58, 11285 (1998). 